\title{
Evaluating the User Experience of a Web-based Child Health Record System
}

\author{
Nadia Abdul Wahab ${ }^{1 *}$, Nor Marlina Sahabudin ${ }^{2}$, Aznoora Osman ${ }^{3}$, Norfiza Ibrahim ${ }^{4}$, \\ ${ }^{1,2,3,4}$ Faculty of Computer and Mathematical Sciences, \\ Universiti Teknologi MARA, Perlis Branch, Arau Campus, 02600 Arau, Perlis, Malaysia \\ Corresponding author: *nadiawahab@uitm.edu.my \\ Received Date: 2 October 2020 \\ Accepted Date: 15 October 2020
}

\begin{abstract}
The aim for this research is to design, develop and evaluate a web-based child health record system with appointment notification. This system has the ability to record all the medical information about a child such as the height, weight, treatments and medical conditions. It also has the ability to record the visits and immunization details, reminder for next visits and immunization appointments. A User Experience (UX) Test were conducted during the study to evaluate the system. User Experience Questionnaire (UEQ) is the instrument that is being utilized during the UX Testing to evaluate the user experience quality of a product. UEQ has been divided into six scales which are attractiveness, perspicuity, efficiency, dependability, stimulation and novelty. During the UX Testing, the users were given a chance to explore and evaluate the system. The result from the UX Testing indicates that most of the users agree that the system is attractive and has a good perspicuity, efficiency, dependability, stimulation and novelty. In conclusion, this research has achieved the objectives which are to design, develop and evaluate a WebBased Child Health Record System with Appointment Notifications.
\end{abstract}

Keywords: User Experience, UX Testing, User Experience Questionnaire (UEQ), Child Health Record System,

\section{INTRODUCTION}

Every new born baby in Malaysia will be provided with the health record booklet which will be utilized by the medical staffs and kept by the parents. This health record booklet is very important because it records information about the children's health conditions, growth, clinic visits, and appointments with medical staffs, immunization record and guide for parent on how to take care of their children (World Health Organization, 2016). The health record is very important for the parents and medical staffs to access the medical information of a child (Garba, 2016).

However, there are some issues with the manual health record booklet. In order to record the information, the medical staffs need to document it manually in two copies; one for parents and the other one for the medical department. This is very time consuming and could lead to data inconsistency (Ariffin, 2017). The second issue is the booklet is not efficient and effective to be use as it could be missing or damage. This could also lead to privacy issue such as stealing of personal data such as medical information, phone number, identity card number and address from the booklet. Besides that, problems like forgetting the appointments might also happen as there is no mechanism to remind the parents other than from referring to the booklet (Thomas, Beh, \& Nordin, 2019).

Therefore, this project aims to design, develop and evaluate a Web-Based Child Health Record System with Appointment Notification for keeping health record of the children. This system has the ability to 
record all the medical information about the children such as height, weight, treatments and medical conditions. It also has the ability to record visit details, immunization details, and reminder for next visits and immunization appointments.

\section{THE WEB-BASED HEALTH RECORD SYSTEM}

The Web-Based Child Health Record System involves two main users which are medical staffs and parents. Medical staff can register health record for new born baby, update their profile such as address, phone number and view details of the children that have been registered. They also have ability to record medical information of children such as date of birth, type of birth, weight, and height and immunization record in this system. Medical staff also has ability to set an appointment for immunization and visits on the system. Parents have ability to view their children's health information, update their basic profile such as address, phone number and e-mail. They are also able to get reminder about the next appointment for immunizations or clinic visits.

Figure 1 shows the screenshot of the Parents Module. By using this module, parents may view and update their profile, view information about their children such antenatal, medical records, postnatal, birth record and appointment on visits and immunization.

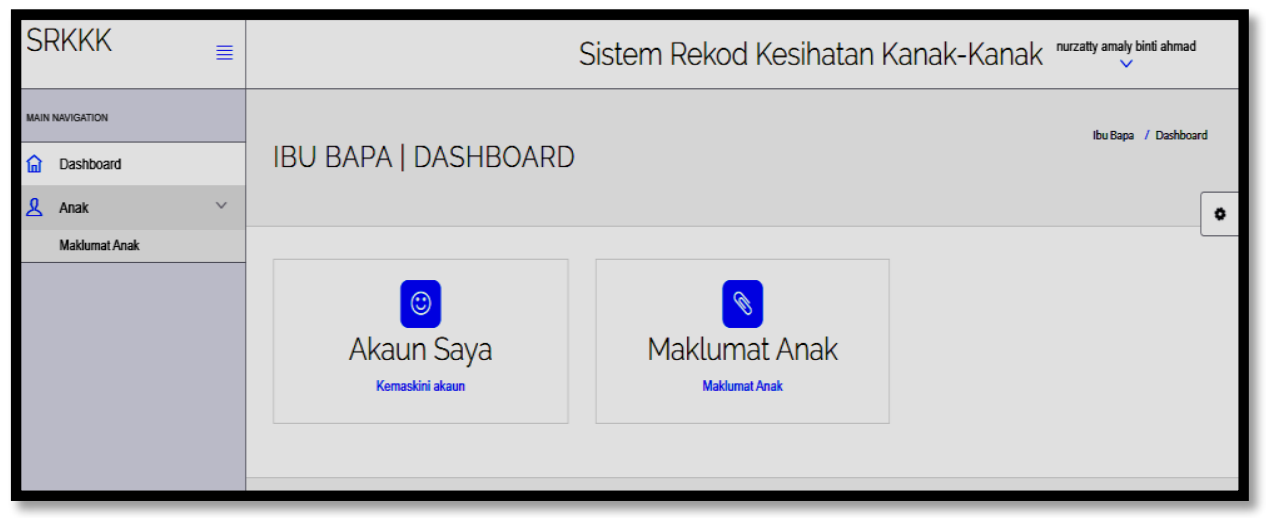

Figure 1: Screenshot of Parents Module

In the Medical Staffs Module (Figure 2), the staffs may view and update their own profile. They also may view information about the parents and children. This module also provides details about visit appointments with the parents and their children.

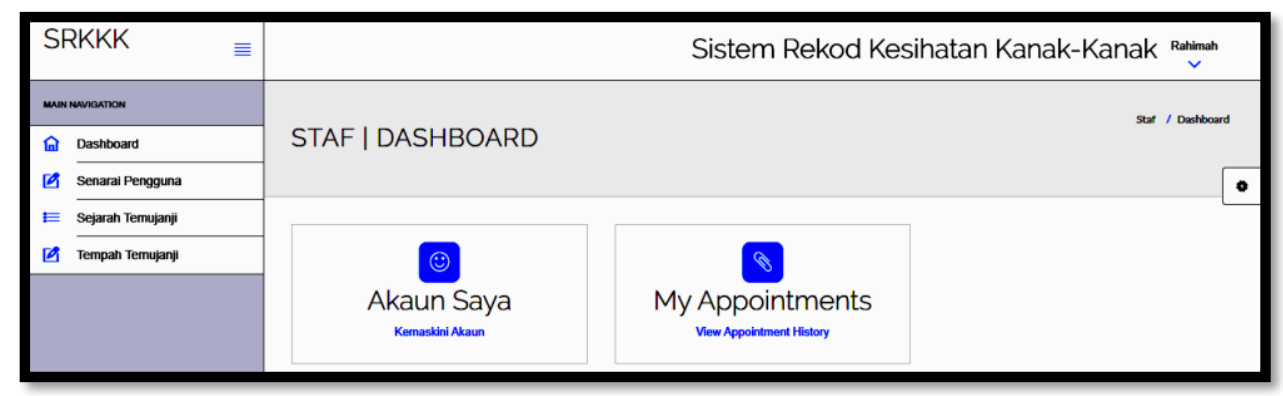

Figure 2: Screenshot of Medical Staff Page 
Figure 3 shows the screenshot of the Visit Record Page. In this page, medical staff may add record on each clinic visit such as date, time, age, purpose of visit, height, weight, head circumstance and other important medical information of a child.

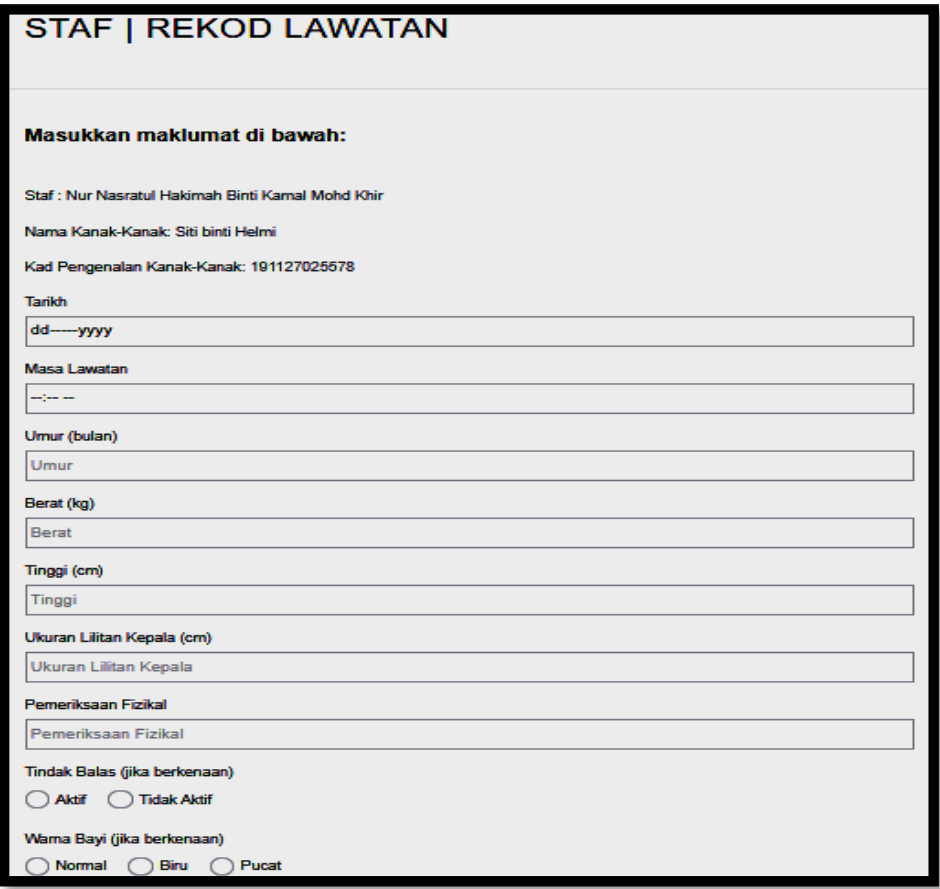

Figure 3: Screenshot of the Visit Record Page

Figure 4 shows the screenshot of the Immunization Record Page. From this page, parents may view and print all of the report on immunization that has been given to their child such as date, type and expiry date of the vaccine. 


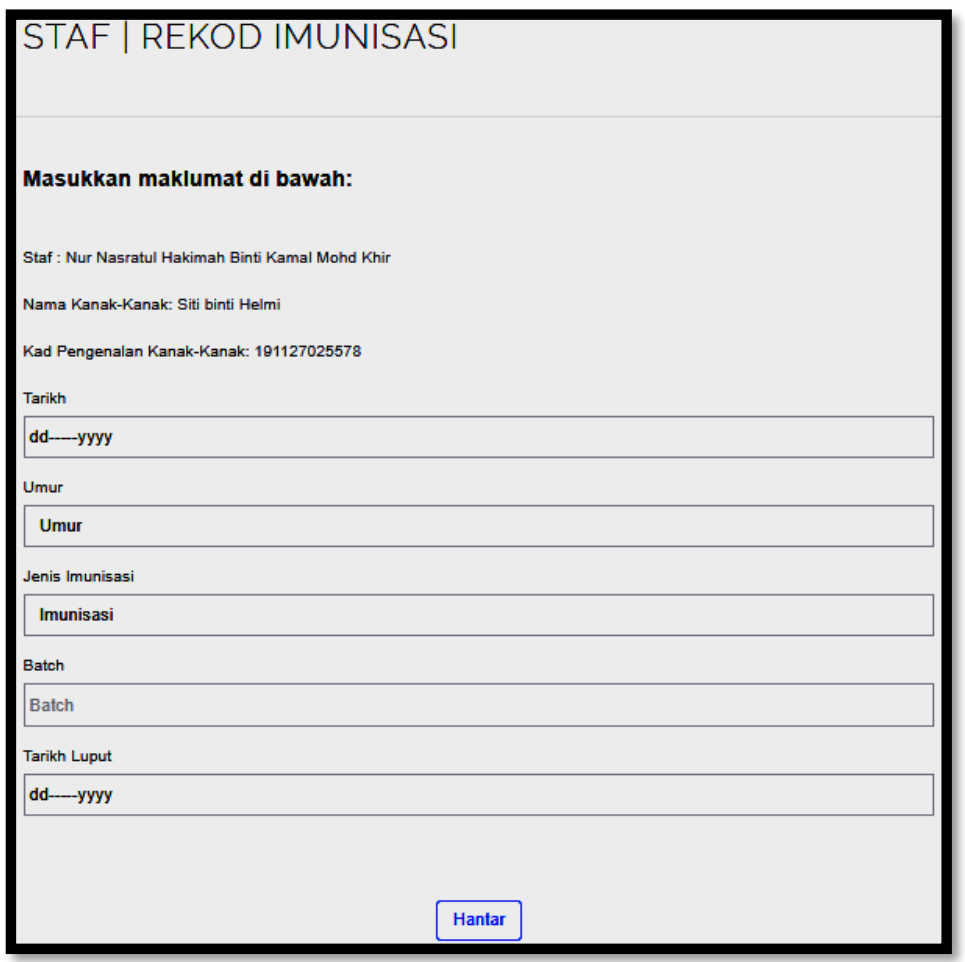

Figure 4: Screenshot of Immunization Page

Figure 5 shows the screenshot of Appointment Details Page. Within this page, the medical staffs may set an appointment and reminder with the parents. The information that need to be recorded are phone number, date and time for the next appointment, purpose, location and also date for send the reminder. Figure 6 shows the screenshot of the Short Messaging System (SMS) reminder that has been sent to the parents automatically from the system.

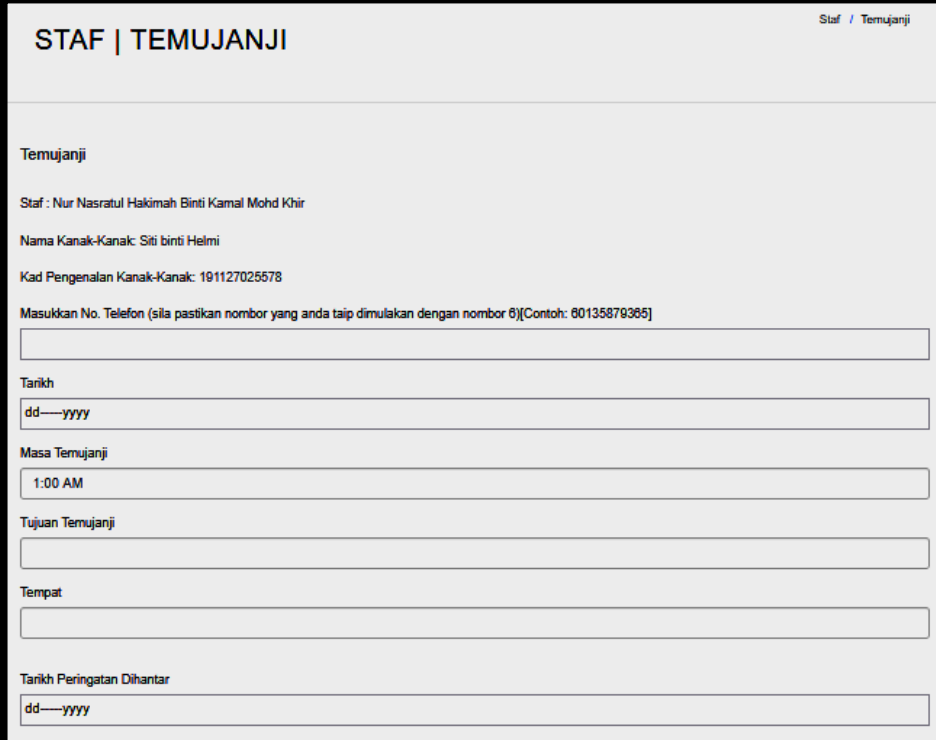

Figure 5: Screenshot of Appointment Details Page 
RMO KMParcel: Anda mempunyai

temujanji pada 2019-11-26 pukul 7:15

AM di Hospital Baling. Sila pastikan

anda datang tepat pada waktu yang

telah ditetapkan.

Nov 25, 7:29 AM • via MY CELCOM

Figure 6: Screenshot of the SMS Reminder

\section{RESULTS AND FINDINGS}

User Experience (UX) Testing is utilized in this study to assess the user emotions, understanding and expectations (Garrets, 2016) towards the Web-Based Child Health Record System. The testing has been conducted with 35 users consisting of $13(37.1 \%)$ medical staffs and $22(62.9 \%)$ parents. During the UX Testing, the users were given a chance to explore and evaluate the system. A questionnaire regarding the user experience aspects of this site is given after system exploration so that all users are able to evaluate the system.

User Experience Questionnaire (UEQ) is the instrument that is being utilized during the UX Testing to evaluate the user experience (UX) quality of a product. The questionnaire consists of twenty-six (26) items related to user's experience while using the system. UEQ has been divided into six scales which are attractiveness, perspicuity, efficiency, dependability, stimulation and novelty (Schrepp, Hinderks, \& Thomaschewski, 2014). The items in UEQ can be measured by using the scale value. The range of the scale is between -3 which indicates very bad and +3 which represents very good (Figure 7).

Schrepp, Hinderks and Thomaschewski (2017) developed a comparison benchmark for UEQ that assist researcher to interpret UX evaluations of a system. The benchmark was created for the UEQ by collecting data from 246 product evaluations using the UEQ. These evaluated products cover a wide range of applications (Schrepp, Hinderks \& Thomaschewski, 2017). Table 1 shows how the categories relate to observed mean scale values in UEQ.

Table 1: Benchmark Intervals for the UEQ Scales

\begin{tabular}{|l|c|c|c|c|c|c|}
\hline & Attractiveness & Efficiency & Perspicuity & Dependability & Stimulation & Novelty \\
\hline Excellent & $\geq 1.75$ & $\geq 1.78$ & $\geq 1.9$ & $\geq 1.65$ & $\geq 1.55$ & $\geq 1.4$ \\
\hline Good & $\geq 1.52$ & $\geq 1.47$ & $\geq 1.56$ & $\geq 1.48$ & $\geq 1.31$ & $\geq 1.05$ \\
& $<1.75$ & $<1.78$ & $<1.9$ & $<1.65$ & $<1.55$ & $<1.4$ \\
\hline Above & $\geq 1.17$ & $\geq 0.98$ & $\geq 1.08$ & $\geq 1.14$ & $\geq 0.99$ & $\geq 0.71$ \\
average & $<1.52$ & $<1.47$ & $<1.56$ & $<1.48$ & $<1.31$ & $<1.05$ \\
\hline Below & $\geq 0.7$ & $\geq 0.54$ & $\geq 0.64$ & $\geq 0.78$ & $\geq 0.5$ & $\geq 0.3$ \\
average & $<1.17$ & $<0.98$ & $<1.08$ & $<1.14$ & $<0.99$ & $<0.71$ \\
\hline Bad & $<0.7$ & $<0.54$ & $<0.64$ & $<0.78$ & $<0.5$ & $<0.3$ \\
\hline \multicolumn{7}{|c|}{ (Source: Schrepp, Hinderks \& Thomaschewski, 2017) } \\
\end{tabular}


Table 2 and Figure 9 shows the mean score obtained from the UX Testing. The result for every item shows that the scale value is above 0 where it represents a positive evaluation. Figure 8 shows the mean score and comparison to benchmark per item for UEQ. From the result, the researcher found that almost all of the users agreed that the system is attractiveness, has a good perspicuity, efficiency, dependability, stimulation and novelty.

Attractiveness scale rates the overall aesthetics of the web-based system and how attracted users are by it. From the UX Test it was found that this system is attractive. The mean score for attractiveness scale of this system is 1.551. This shows that this system is enjoyable, good and pleasing and also friendly. Another scale for UEQ is perspicuity. Perspicuity indicates how easy people understand the system. The mean score for this scale is 1.486 . This shows that, the perspicuity of this system is above average where the system is quite easy to learn, not complicated and not confusing to the users.

Efficiency scales' mean score for this system is 1.507 which indicates that this system has a good efficiency. This shows that by using this system the users could solve their tasks without unnecessary effort and the system react fast to user input. Dependability scale gives an idea about how the system seeming trustworthy. The result from the UX Testing shows that this system has a good dependability with the mean score of 1.500 . This indicates that this system is predictable, supportive, secured and meets the users' expectations.

The joy of use is measured within the stimulation scale and the mean score for this scale is 1.438 . This demonstrates that this system is has a good value, interesting and motivating. Novelty scale in the UEQ represents how innovative a tool is perceived. The results from the UX Test shows that this system has a good novelty with the mean score of 1.349. This indicates that the users feel that this system is innovative, creative and inventive.

Table 2: The Value of Mean and Variance for UEQ Scales

\begin{tabular}{|l|c|c|c|}
\hline UEQ Scales & Mean Score & Variance & $\begin{array}{c}\text { Comparison to } \\
\text { Benchmark }\end{array}$ \\
\hline Attractiveness & 1.551 & 0.51 & Good \\
\hline Perspicuity & 1.486 & 0.51 & Above Average \\
\hline Efficiency & 1.507 & 0.43 & Good \\
\hline Dependability & 1.500 & 0.47 & Good \\
\hline Stimulation & 1.438 & 0.44 & Good \\
\hline Novelty & 1.340 & 0.39 & Good \\
\hline
\end{tabular}




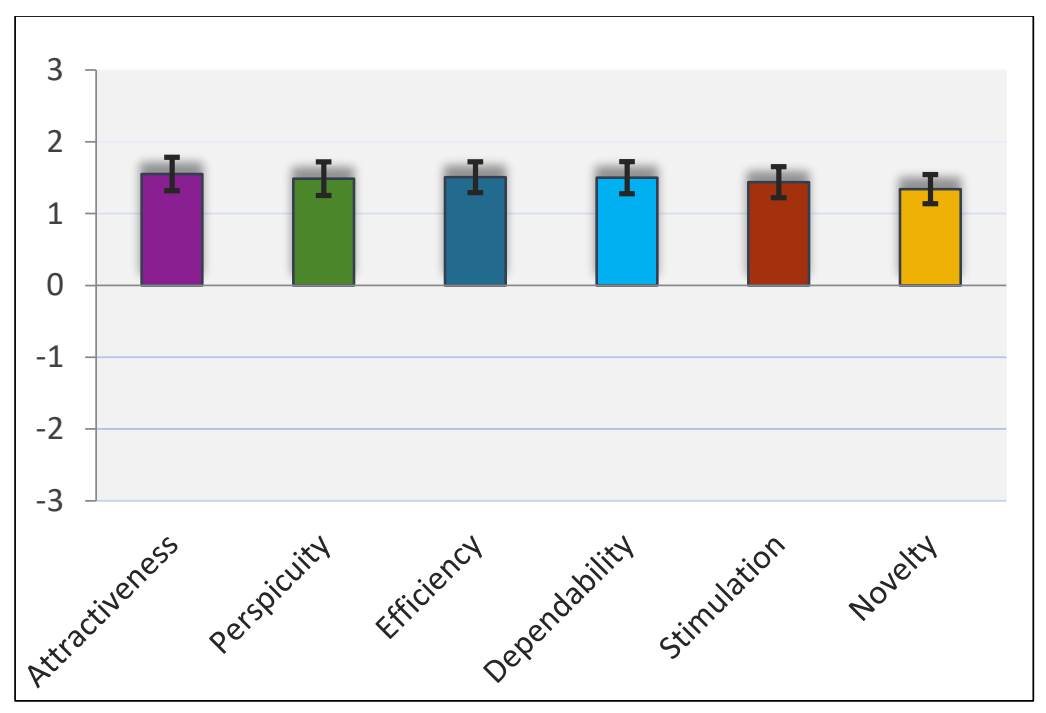

Figure 7: Mean Score for UEQ Scales

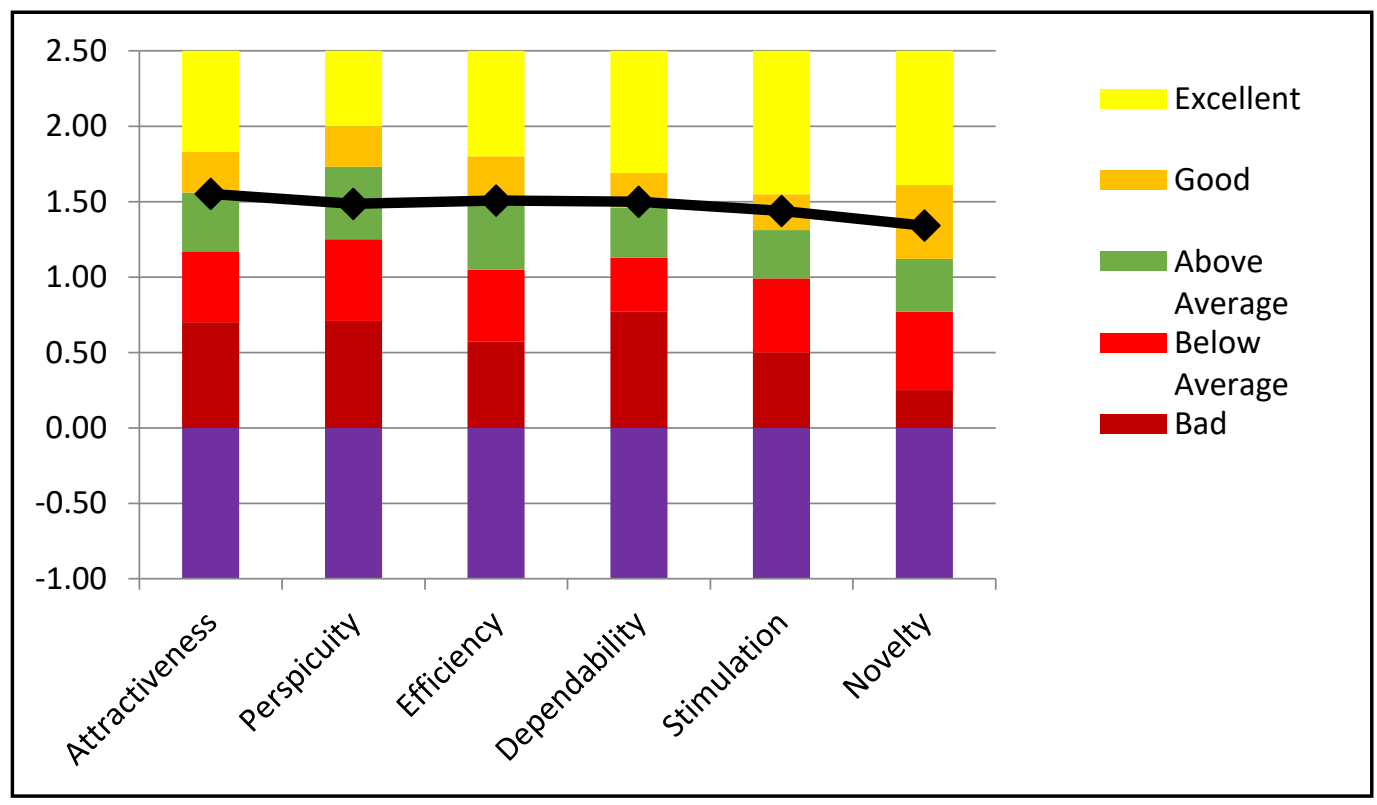

Figure 8: Mean Score and Comparison to Benchmark for UEQ Scales

\section{CONCLUSION AND RECOMMENDATION}

This project aims to design, develop and evaluate a Web-Based Child Health Record System with Appointment Notification for keeping health record of the children. This system has the ability to record all the medical information about the children such as height, weight, medical conditions and so on. It also has the ability to record visit details, immunization details, reminder for visit and immunization appointments. This system involves two main users which are medical staffs and parents.

User Experience (UX) Testing is utilized in this study to assess the user emotions, understanding and expectations towards the Web-Based Child Health Record System. The result from the UX Testing indicates that most of the users had a good experience while using this system. Most of the users are in 
the opinion that this system is attractiveness, has a good perspicuity, efficiency, dependability, stimulation and novelty. Most of them also would like to recommend this application to be used in the near future as a system to record all the medical information about the children in clinics or hospitals.

For future works, there are few recommendations and suggestions that can be implemented to enhance this study. As smart phones are becoming very popular nowadays, this system could also be developed as a mobile app to encourage more users. More modules could be introduced to the system such as parental guide, growth tracking and diet recommendation for children based on their medical conditions. More respondents especially the medical staffs should participate during the testing so that the researcher will get more opinions and views that can be utilized in improving the system especially on the usability aspect.

\section{REFERENCES}

Ariffin, W. (2017). Child health in Malaysia. Malaysia Health, 18(6), 17. https://doi.org/10.1007/978-94007-0753-5_100449

Garba, K. D. (2016). Significance and Challenges of Medical Records: A Systematic Literature Review. Retrieved from https://www.researchgate.net/publication/309034245

Garrett, J. J. (2016). Customer Loyalty and the Elements of User Experience. Management Review, 3540.

Schrepp, M., Hinderks, A., \& Thomaschewski, J. (2014, June). Applying the user experience questionnaire (UEQ) in different evaluation scenarios. In International Conference of Design, User Experience, and Usability (pp. 383-392). Springer, Cham.

Schrepp, M., Hinderks, A., \& Thomaschewski, J. (2017). Construction of a Benchmark for the User Experience Questionnaire (UEQ). International Journal of Interactive Multimedia and Artificial Intelligence, 4(4), 40. https://doi.org/10.9781/ijimai.2017.445

Thomas, S., Beh, L., \& Nordin, R. Bin. (2019). Health care delivery in Malaysia: changes, challenges and champions. 2(2), 1-10. https://doi.org/10.4081/jphia.2011.e23

World Health Organization. (2016). Medical Records Manual : A Guide for Developing Countries. 